Acta Technologica Agriculturae 4

Nitra, Slovaca Universitas Agriculturae Nitriae, 2017, pp. 96-103

\title{
THEORY OF VIBRATIONS OF SUGAR BEET LEAF HARVESTER FRONT-MOUNTED ON UNIVERSAL TRACTOR
}

\author{
Volodymyr BULGAKOV', Valerii ADAMCHUK ${ }^{2}$, Ladislav NOZDROVICKÝ ${ }^{3 *}$, Yevhen IHNATIEV ${ }^{4}$ \\ ${ }^{1}$ National University of Life and Environmental Sciences of Ukraine \\ ${ }^{2}$ National Scientific Centre "Institute for Agricultural Engineering and Electrification" of the National Academy \\ of Agrarian Sciences of Ukraine \\ ${ }^{3}$ Slovak University of Agriculture in Nitra, Slovak Republic \\ ${ }^{4}$ Tavria State Agrotechnological University of Ukraine
}

\begin{abstract}
The harvest and transport of sugar beet leaves during harvesting can be considered a current task in the area of sugar beet growing system development. A rotary sugar beet leaf cutting mechanism is used for achieving the significant increase in the harvester forward speed during harvest. This leads to intensive vibrations of the topping mechanism in the longitudinal vertical plane causing the decrease of harvest quality. Therefore, it is necessary to analytically determine the effect of kinematic and design parameters of the sugar beet topping mechanism front-mounted on the tractor and to discover the value of the amplitude of oscillations in the longitudinal vertical plane of its sugar beet topping mechanism. We have constructed a mathematical model of the estimated motion of this machine by means of dynamics equations in Lagrange Il-kind form. A system consisting of two non-linear differential equations was obtained by developing the equivalent scheme of selected generalized coordinates and by performing the necessary mathematical transformations. This system describes the vibrations of the sugar beet topping mechanism in the longitudinal vertical plane. The optimal design and kinematic parameters of the sugar beet topper mechanism front-mounted on the tractor were determined by means of our own software and numerical solution of the differential equation system, which allows the reduction of mentioned vibrations.
\end{abstract}

Keywords: sugar beet; beet tops; rotary cutting mechanism; universal wheel-type tractor; vibrations; differential equations

High-efficiency capacity and qualitative harvest of sugar beet leaves can be considered a sufficiently complex and up-to-date task for the sugar beet production field. In recent years, the multi-phase technology of sugar beet leaf harvesting became the most used method around the world. In the starting phase, there is provided a smooth basic cut of all sugar beet tops. In the next phase, there is individual sensing of sugar beet heads in the row and subsequent head cleaning or secondary topping of sugar beet heads from leaf residues.

The abovementioned operations are carried out consistently for sugar beet roots located in the soil and leaf harvest is preceded by digging out of sugar beet roots from the soil. Therefore, it is important to take into account that sugar beet toppers are front-mounted in relation to the carrying machine. However, on the base of conducted experimental studies, it was found that during operation, the tractor front-mounted topping machine carries out movement in space, and all this movement is determined by the field surface relief, forward speed of the tractor, location of sensing wheels with respect to the suspension system of the machine, etc. All the mentioned factors significantly affect the quality of this technological process.
If pneumatic wheels are used as sensing wheels, the vibrations of the sugar beet topping harvester occur on vertical plane, affecting the quality of the technological process. Regardless of the wide spread of front-mounted modules of the sugar beet leaf harvesters manufactured in western countries, as well as of some domestic solutions of front-mounted sugar beet leaf harvesters, the analytical investigation of their vibrating motion is not affected.

In order to improve the efficiency of research, it is very important to use the adequate research tools. According to Bulgakov and Korenko (2007), a mathematical model describing the process of sugar beet direct extraction from soil can be used; however, in case of the sugar beet leaf harvester front-mounted on the universal tractor, such a model was not developed and it remains a goal for future research. However, using the procedures described by Vasilenko (1996), it is possible to develop a mathematical model for the calculation of this machine, which will make it possible to investigate the effects of its design parameters on vibrations along the rows of sugar beet roots and the soil surface unevenness.

Multiple scientific papers have dealt with the work of the sugar beet topping mechanism with modern design. These papers have analysed the design and technical specifications 
of different sugar beet topping harvesters (Lammers et al., 2010) as well as sugar beet topper modules, which are the part of sugar beet harvesters (Lilleboe, 2014). Harvesting of root crops creates specific requirements on the function of used mechanisms. Such requirements are analysed in relation to the root vegetable, and according to Jobbágy et al. (2011), during the harvest of root crops, it is very important to consider the variability of agro-physical properties of the harvested root crop.

This significantly and unacceptably pollutes the roots of sugar beets when they are subsequently excavated from the soil (Huijbregts et al., 2013), which significantly reduces the yield of sugar. Therefore, a part of the research is devoted to justifying the rational height of the cut and determining the damage degree to sugar beet root heads (Ihnatiev, 2016). Such results have been published by Bulgakov et al. (2016). However, research results containing general and complete theories which would allow determining the vibrations of the cutting mechanism are currently missing.

Within our research activity, the main objective was to determine the effect of kinematic and design parameters of the sugar beet leaf harvester front-mounted on wheeltype tractor and to observe the value of the amplitude of oscillations in the longitudinal vertical plane of the sugar beet topping mechanism.

\section{Material and methods}

In order to achieve the abovementioned goals and objectives, computational methods for calculation of mathematical models of agricultural machines and machine units functioning were used, based on theoretical mechanics, higher mathematics, programming methods and numerical solution of differential equation systems by means of PC. Thus, in particular, when constructing an equivalent scheme for the motion of the front-mounted sugar beet leaf harvesting machine, the fact that its sensing wheels move along the uneven surface must be taken into consideration; this unevenness was approximated by a harmonic function.
The copying wheels themselves were modelled in the form of elasticdamping models reflecting their elastic and viscous properties. This provides possibilities to apply the investigating methods of complex oscillatory systems and to use two sources of kinematic excitation - unevenness of the soil surface and pneumatic tyres of sensing wheels.

In the next step, by means of the methods of analytical mechanics, there were chosen the generalized coordinates of the considered oscillatory system, which made it possible to compile a system of nonlinear differential equations describing the oscillations of the sugar beet leaf harvesting machine mass centre and the knife ends of its rotary leaf cutting working organ.

Using the method of solving differential equations, first, the solution of the resulting system of differential equations in closed form was implemented, followed by RungeKutta numerical methods in the MathCAD system.

\section{Results and discussion}

Within our research, a new universal sugar beet leaf harvester has been developed, carrying out the technological processes of sugar beet leaf harvest based on the principle of the mower-crusher front-mounted on the wheel-type universal tractor.
Figure 1 shows the constructive and technological scheme of the machine and the tractor unit, which provides a cut of beet tops and transports all the cut mass in the load compartment of a transport vehicle which is moving along it.

The technological process of harvesting of sugar beet tops runs as follows: during the motion of the universal wheel-type tractor (equipped with narrow tyres) along the rows of the sugar beet, the pneumatic sensing wheels [3], located in the front part of the movable frame [1], are adjusted in such a way that the used rotary sugar beet leaf cutting mechanism [4], equipped with blades, allows reaching the required height of cut. The knives have an arcuate shape and are pivotally mounted on the cylindrical generatrix along the length of the rotor [4].

Leaves cut by arcuate knives are moved upwards, then they fall into the screw conveyor, which transports the crop material to the frontal part of the machine. In the next step, the transporting working organ [5] unloads the crop material into the body of the transport unit moving alongside the sugar beet leaf harvester by means of mechanism [6].

The drive [7] of the sugar beet leaf harvester is provided from the front PTO mounted on the used tractor.

The fact that the rotary type sugar beet leaf harvester is having only one working organ - rotor with blades can be considered a main advantage of this machine.

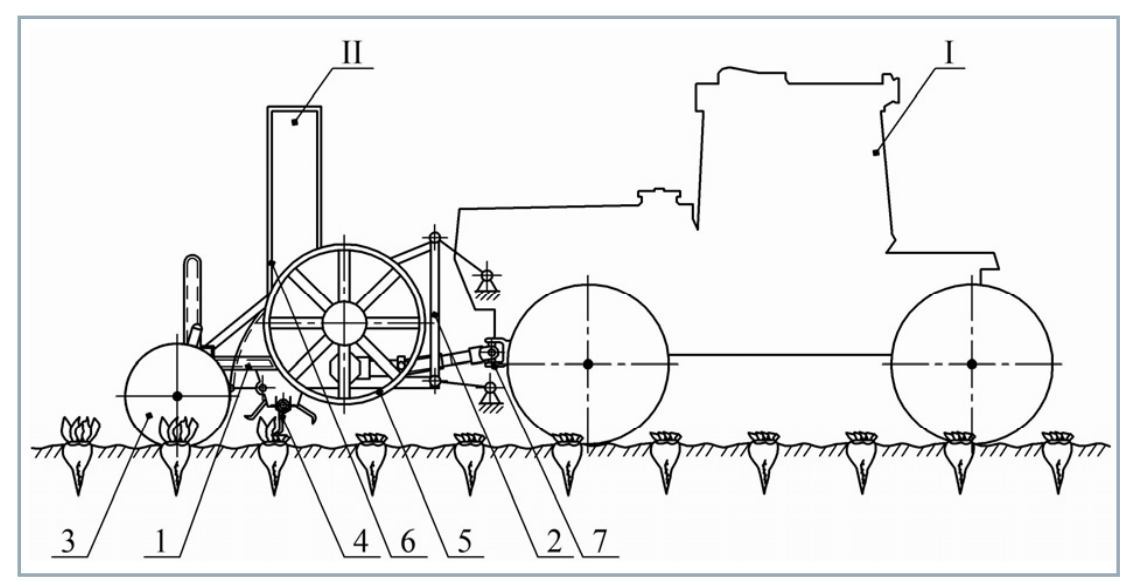

Fig. 1 Structural and technological scheme of the machine and tractor unit for harvesting of sugar beet leaves:

I - universal wheel-type tractor; II - front-mounted sugar beet leaf harvest machine:

1 - frame; 2 - front-mounted hitch; 3 - supporting pneumatic sensing wheel; 4 - rotary leaf cutting mechanism; 5 - conveying working mechanism; 6 - stabilizing mechanism;

7 - drive of the machine from the front-mounted PTO of the tractor 


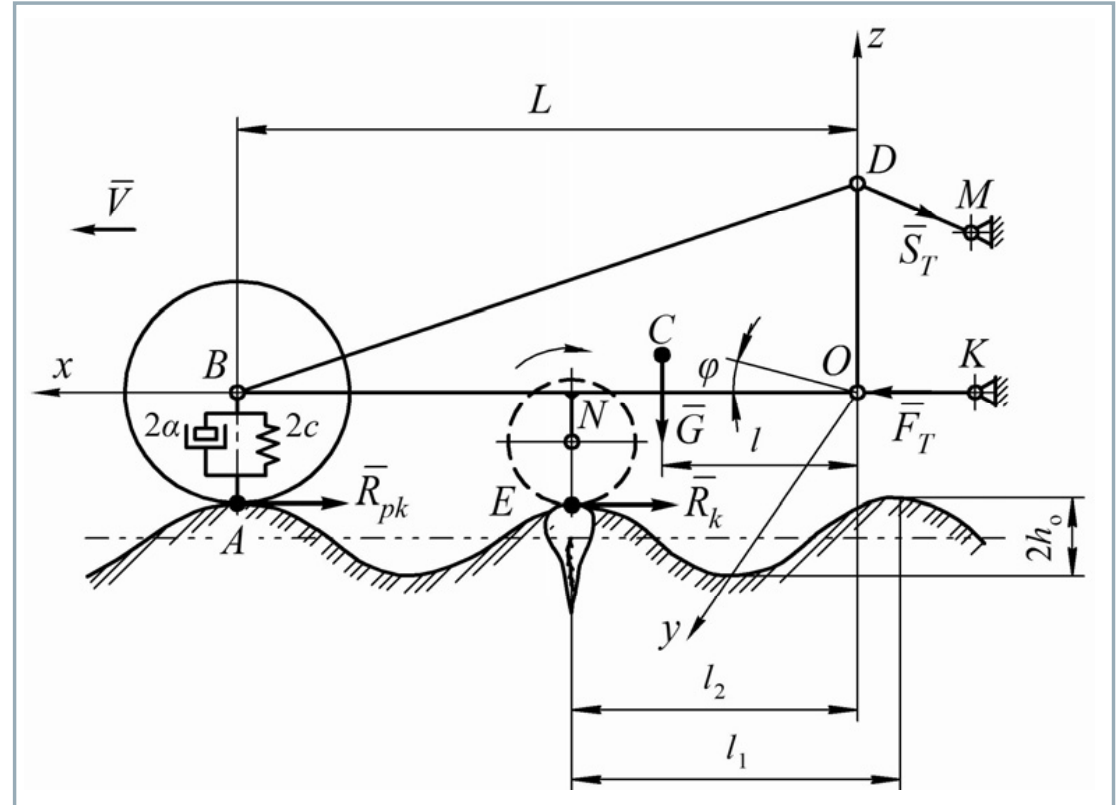

Fig. 2 Equivalent scheme of the sugar beet leaf harvester front-mounted on a universal tractor

Regardless of the fact that both the mechanical and technological bases of the cutting process provided by sensing-less cutting without support (Bosoj et al., 1978), as well as sugar beet leaf harvest (Khvostov and Reingart, 1995) are widely studied and investigated, there is a necessity to perform further research of the given technological process.

In order to determine the impacts of design and kinematic parameters of the sugar beet leaf harvester frontmounted on a universal wheel-type tractor on a magnitude value of the oscillation amplitude in a longitudinal vertical plane of the leaf cutting mechanism, it was necessary to develop a mathematical model of it.

We have developed a primarily equivalent scheme of movement of the sugar beet leaf harvester frontmounted on a universal wheel-type tractor in the longitudinal vertical plane (Fig. 2).

As it can be seen in Fig. 2, the sugar beet leaf harvester is connected to the tractor by means of two lower draw-bars $O K$ and one upper draw-bar $D M$, with hinges in the points $O, D, M$ and $K$. Radiuses of the sensing (gauge) wheels and leaf cutting mechanism are marked respectively as $R$ and $R_{1}$. The weight of the whole sugar beet leaf harvester is marked as $M$; the weight of the two sensing (gauge) wheels is determined by means of $m=m_{1}+m_{2}$ (where $m_{1}$ - weight of the first sensing (gauge) wheel, $m_{2}$ - weight of the second sensing (gauge) wheel). The weight $m$ of both sensing (gauge) wheels is concentrated in the point $B$. The gravity force of the sugar beet leaf harvester which is affecting its mass centre (point $C$ ) - is marked as $\bar{G}$.

We assign a given dynamical system to a fixed Cartesian coordinates XOYZ. In such a case, the plane $X O Z$ coincides with the longitudinal plane of the machine - sugar beet leaf harvester and can be considered a vertical plane in relation to the field surface.

Pneumatic sensing (gauge) wheels are shown in the form of elastic-damping models, which have a combined total spring constant $2 c$ and damping coefficient $2 \alpha$ (Kutkov, 2004).

In general, we consider that the sensing (gauge) wheels are sensing upper (more loose) surface layer of the soil when moving between rows of the sugar beet; however, they still move over the soil surface unevenness, which has a longitudinal profile close to a sinusoidal type.

In the first approximation, the pneumatic sensing (gauge) wheel, moving in inter-row space of the sugar beet and sensing upper loosen soil layer, can be considered in contact with the soil surface unevenness in the point $A$. Such soil surface unevennesses (in smoothed form) can be presented by a harmonic function, i.e. analytical expression of this type (Morozov and Gringauz, 1969): $h=h_{\circ}\left(1-\cos \frac{2 \pi x}{l_{1}}\right)$, where $h$ - ordinate of the height of soil surface unevenness, $\mathrm{m} ; h_{o}$ - one half of the height of soil surface unevenness, $m ; l_{1}$ - the step of soil surface unevenness, $m ; m=$ $V t$ - current coordinate, $\mathrm{m} ; \mathrm{V}$ - forward speed of the sugar beet leaf harvester, $\mathrm{m} \cdot \mathrm{s}^{-1}$.

Thanks to its weight, the tractor provides the sensing of the upper soil layer by its wheels when passing over the soil surface unevenness more significantly, thereby it smoothes out the existing soil surface unevenness. This results in the reduction of amplitudes of vertical vibrations of the tractor gravity centre.

Obviously, for these reasons, the vertical oscillations of the wheel-type tractor are significantly reduced; however, it should be assumed that they do not disappear.

Therefore, the hinges in points $K$ and $M$ (Fig. 2), as belonging to the tractor, also perform vertical vibration. However, thanks to the hinges in the points $O$ and $D$ and a large mass of the sugar beet leaf harvester, all these vibrations are not practically transferred to the harvester.

Vibrations of the used tractor are causing just angle vibrations of the draw-bars $O K$ and $D M$ which are connecting the sugar beet leaf harvester to the tractor. Practically, it can be taken into account that hinges $O$ and $D$ do not vibrate in vertical plane, and the draw-bars $O K$ and $D M$ are turning around the points $O$ and $D$ respectively, while other end points (hinges $K$ and $M$ ) are vibrating independently together with the tractor. Based on abovementioned facts, the sugar beet leaf harvester connection point (hinges $O$ and D) can be considered moving straightforwardly and uniformly in the first approximation.

As a sugar beet leaf harvester is front-mounted on a tractor, therefore its sensing (gauge) wheels primarily perceive the soil surface unevenness and that is the cause of the vertical angular oscillation of sugar beet leaf harvester frame around the point $O$.

Obviously, the rotation angle $\varphi$ of the machine frame around the point $O$ depends essentially on the 
value $h$ of unevenness at the point $A$ at which the gauge wheel occurs in the given moment. Therefore, this certain angle $\varphi$ in some approximations can be determined from the following expression: $\varphi=\frac{h}{L}$, where $L$ - distance from the point $B$ of the axis of the sensing (gauge) wheel to the suspension $O$ of the frame to the lower draw-bar $K$ (Fig. 2).

Taking into account the $h=h_{o}\left(1-\cos \frac{2 \pi x}{l_{1}}\right)$, we obtain the final value of angle $\varphi$ :

$$
\varphi=\frac{h_{o}}{L}\left(1-\cos \frac{2 \pi x}{l_{1}}\right)
$$

Thus, the surface unevenness of the field can be considered a kinematic angular oscillation exciter of the sugar beet leaf harvester frame. However, the turning of the machine frame around the point $O$ can be provided only by a moment of certain force, the line of action of which passes through the point $O$.

In this case, such a force is generated by this unevenness of soil surface, which will obviously vary in accordance with the sinusoidal law that is valid also for the unevenness of the soil surface profile of the field, i.e. according to the following equation:

$$
H=H_{o}\left(1-\cos \frac{2 \pi x}{l_{1}}\right)
$$

where:

$H_{o} \quad$ - the amplitude of the mentioned force

Obviously, this force is applied in the point $A$, directed along the axis $O Z$ and in relation to the point $O$, its moment will be equal to:

$$
M_{o}(H)=H_{o} L\left(1-\cos \frac{2 \pi x}{l_{1}}\right)
$$

Mentioned force can be considered external force acting on the frame of sugar beet leaf harvester from field surface.

In addition to this main force, horizontal reaction $\bar{R}_{p k}$ is applied in the point $A$, which is also, generally speaking, a variable of great importance for the moving sensing (gauge) wheel up the sinusoid and less - when moving the sensing (gauge) wheel down according to the said sinusoid. Also, the reaction $\bar{R}_{k}$ of shear resistance of the rotary sugar beet leaf cutting mechanism acts in the point $E$.

Obviously, these two forces play an insignificant role in establishing the angular oscillations of the machine frame in comparison to force $\bar{H}$ and weight $\bar{G}$ of the sugar beet leaf harvester machine itself.

Forces $\bar{R}_{p k}$ and $\bar{R}_{k}$ primarily provide resistance to movement of the sugar beet leaf harvester on the field and they are directed oppositely in relation to driving force $\bar{F}_{T}$ from the side of the used tractor.

The force $\bar{S}_{T}$ of the tension of draw-bar DM also does not play any significant role in creation of angle vibrations of the frame of sugar beet leaf harvester due to its low value. In general, driving force $\bar{F}_{T}$ crosses the point $O$, and thus it only pushes the sugar beet leaf harvester forward without creating any moment of rotation of the sugar beet leaf harvester frame around the point $O$.

Thus, an essential role in the creation of angular oscillation of the sugar beet leaf harvester frame in vertical plane is played only by force $\bar{H}$ (kinematic excitation), arising due to the presence of unevenness of field soil surface, and force $\bar{G}$ caused by the weight of the sugar beet leaf harvester. It should be noted that the action line of mentioned forces coincides with the direction of movement of the application points, i.e. the points $A$ and $C$ respectively.

However, it should be noted that the amplitude $H_{0}$ of force $\bar{H}$, as well as reactions $\bar{R}_{p k}$ and $\bar{R}_{k}$, are unknown variables. Therefore, it is impossible to take advantage of the fundamental law of dynamics for the preparation of differential equations of motion of the sugar beet leaf harvester front-mounted on wheel-type tractor with regard to soil surface unevenness. To solve this issue, it is advisable to take advantage of differential equations of motion in the form of Lagrange II (Vasilenko, 1996).

First of all, the generalized coordinates of the dynamic system must be defined. The sugar beet leaf harvester mass centre (point $C$ ) in the longitudinal vertical plane is completely determined by an independent coordinate $\varphi$.

Since the mass centre of the pneumatic gauge wheels (point $B$ ) performs an independent oscillatory motion due to elastic-damping properties of the sensing (gauge) wheels and the ordinates of elevation of the soil surface unevenness $h$ are significantly smaller less than length $L$, we can assume that these vibrations may be determined by an independent coordinate $Z$. Thus, the considered vibration system can be reduced to two generalized coordinates: $q_{1}=\varphi, q_{2}=Z$.

Considering the above coordinates, the main influence on the vertical vibrations of the sugar beet leaf harvester has the following factors: viscoelastic resistance of the sensing (gauge) wheel tyres, the machine weight and soil surface unevenness size. Thus, it is relevant to believe that only potential forces and viscous resistance are influencing the studied mechanical system.

We use this fact in the preparation of differential motion equations of the dynamical system based on the Lagrange II.

According to Butenin et al. (1985), if only forces acting on the dynamic system are potential forces and forces of viscous resistance, then the generalized forces $Q_{i}$, entering the Lagrange Equation II, can be determined by the following equation:

$$
Q_{i}=-\frac{\partial P}{\partial q_{i}}-\frac{\partial R}{\partial \dot{q}_{i}}
$$

where:

$P \quad$ - potential energy of the dynamic system

$R \quad$ - dissipative function (Relay function)

$q_{i} \quad$ - generalized coordinate

$\dot{q}_{i} \quad$ - generalized speed

In this case, differential equations of Lagrange II have the form as follows:

$$
\frac{d}{d t}\left(\frac{\partial T}{\partial \dot{q}_{i}}\right)-\frac{\partial T}{\partial q_{i}}=-\frac{\partial P}{\partial q_{i}}-\frac{\partial R}{\partial \dot{q}_{i}}
$$


where:

$T \quad$ - kinetic energy of the given dynamic system

The dynamic system observed in this paper is considered to have two degrees of freedom, and therefore, as noted above, two generalized coordinates $q_{1}=\varphi$ and $q_{2}=Z$. Therefore, as a result, we obtain a system of two differential equations in the form of Lagrange II:

$$
\left.\begin{array}{c}
\frac{d}{d t}\left(\frac{\partial T}{\partial \dot{\varphi}}\right)-\frac{\partial T}{\partial \varphi}=-\frac{\partial P}{\partial \varphi}-\frac{\partial R}{\partial \dot{\varphi}} \\
\frac{d}{d t}\left(\frac{\partial T}{\partial \dot{Z}}\right)-\frac{\partial T}{\partial Z}=-\frac{\partial P}{\partial Z}-\frac{\partial R}{\partial \dot{Z}}
\end{array}\right\}
$$

Next, we define the components that are included in the system of equations (6). Thus, the kinetic energy $T$ of the mechanical system consists of the kinetic energy of the sugar beet leaf harvester forward motion, angular movement of the machine frame around point $O$, and the kinetic energy of vertical oscillations of its sensing (gauge) wheels. Therefore, it will be equal to:

$$
T=\frac{M V^{2}}{2}+\frac{I_{\text {oy }} \dot{\varphi}^{2}}{2}+\frac{m \dot{Z}^{2}}{2}
$$

where:

$M \quad$ - weight of the sugar beet leaf harvester, $\mathrm{kg}$

$V \quad$ - forward speed of the machine, $\mathrm{m} \cdot \mathrm{s}^{-1}$

$l_{\text {oy }} \quad$ - moment of inertia of the machine in relation to the axis $O Y, \mathrm{~kg} \cdot \mathrm{m}^{2}$

$m \quad$ - weight of the sensing (gauge) wheels, $\mathrm{kg}$

The potential energy of this dynamic system will be equal to the work of elastic forces of both sensing (gauge) wheel tyres and therefore is determined by the following expression:

$$
P=c(L \varphi-Z)^{2}
$$

where:

c - stiffness coefficient of pneumatic tyres measured by sensing (gauge) wheels, $\mathrm{N} \cdot \mathrm{m}^{-1}$

$L \quad$ - distance from the axis of the hinge of the sugar beet leaf harvester (point $O$ ) to the axis of its sensing (gauge) wheels (point $B$ ), $\mathrm{m}$

The dissipative function $R$ of the above dynamic system is determined by the forces of viscous resistance proportional to the speed of movement, and therefore equal to:

$$
R=\alpha(L \dot{\varphi}-\dot{Z})^{2}
$$

where:

$\alpha \quad$ - the damping coefficient of sensing (gauge) wheels, $\mathrm{N} \cdot \mathrm{s} \cdot \mathrm{m}^{-1}$

The abovementioned resistance forces are also caused by the sensing (gauge) wheel tyres of the sugar beet leaf harvester.

We perform necessary transformations caused by the application of dynamical equations in the form of Lagrange II.
As a result, we finally obtain:

$$
\left.\begin{array}{c}
I_{\text {оу }} \ddot{\varphi}+2 c L(L \varphi-Z)+2 \alpha L(L \dot{\varphi}-\dot{Z})=0, \\
m \ddot{Z}+2 c(Z-L \varphi)-2 \alpha(L \dot{\varphi}-\dot{Z})=0
\end{array}\right\}
$$

The resulting system (10) consisting of two differential equations is modelling the vibrations of the sugar beet leaf harvester mass centre (point $C$ ) in the longitudinal vertical plane and vibrations of the centre of sensing (gauge) wheel (point $B$ ).

The system of equations (10) is transformed to the convenient form as follows:

$$
\left.\begin{array}{c}
\ddot{\varphi}+\frac{2 c L^{2}}{l_{\text {oy }}} \varphi-\frac{2 c L}{l_{\text {oy }}} Z+\frac{2 \alpha L^{2}}{l_{\text {oy }}} \dot{\varphi}-\frac{2 \alpha L}{l_{\text {oy }}} \dot{Z}=0, \\
\ddot{Z}+\frac{2 c}{m} Z-\frac{2 c L}{m} \varphi-\frac{2 \alpha L}{m} \dot{\varphi}+\frac{2 \alpha}{m} \dot{Z}=0
\end{array}\right\}
$$

Thus, with respect to the unknown generalized coordinates $\varphi$ and $Z$, the obtained system of nonlinear differential equations (11) is estimated as a mathematical model of the motion of the sugar beet leaf harvester front-mounted on a universal wheel-type tractor.

The system of differential equations (11) with the initial conditions can be solved by a PC program using adapted Runge-Kutta method in the MathCAD system compiled for this purpose.

However, the resulting mathematical model is quite general. It is suitable for describing the vertical vibrations of the sugar beet leaf harvester front-mounted on a universal wheel-type tractor when the law of changes in soil surface unevenness profile is unknown.

Therefore, in this particular case, expression (1) is the solution of system (11). Substituting this expression into one of the system of equations (11), we obtain the differential equation for determining the law of change of coordinate $Z$.

We substitute the expression (1) to the second differential equation system (11). To do this, we write the expression (1) as follows:

$$
\varphi=\frac{h_{o}}{L}\left(1-\cos \frac{2 \pi V t}{l_{1}}\right)
$$

By differentiating the Eq. (12) with respect to time $t$, we obtain:

$$
\dot{\varphi}=\frac{2 \pi h_{o} V}{L \cdot I_{1}} \cdot \sin \frac{2 \pi V t}{I_{1}}
$$

Substituting the Eqs (12) and (13) to the second equation of system (11), we obtain the following differential equation for the unknown function $Z(t)$ :

$\ddot{Z}+\frac{2 \alpha}{m} \dot{Z}+\frac{2 c}{m} Z=\frac{4 \pi \alpha h_{o} V}{m l_{1}} \sin \frac{2 \pi V t}{l_{1}}-\frac{2 c h_{o}}{m} \cos \frac{2 \pi V t}{l_{1}}+\frac{2 c h_{o}}{m}$

Equation (14) is a linear differential equation of the second order with constant coefficients with the right side. Its general solution, as it is known, consists of a general solution of linear homogeneous equation and a particular solution of inhomogeneous equation, the form of which is determined by the views of the right side of Eq. (14): 


$$
Z=Z_{\text {hom. }}+Z^{*}
$$

In the first step, we find the general $Z_{\text {hom. }}$ solution of the homogeneous equation:

$$
\ddot{Z}+\frac{2 \alpha}{m} \dot{Z}+\frac{2 c}{m} Z=0
$$

The characteristic equation of homogeneous Eq. (16) has the following form:

$$
k^{2}+\frac{2 \alpha}{m} k+\frac{2 c}{m}=0
$$

The bases of the above characteristic equation will take the following value:

$$
\begin{aligned}
& k_{1}=-\frac{\alpha}{m}+\sqrt{\frac{\alpha^{2}}{m^{2}}-\frac{2 c}{m}} \\
& k_{2}=-\frac{\alpha}{m}-\sqrt{\frac{\alpha^{2}}{m^{2}}-\frac{2 c}{m}}
\end{aligned}
$$

Due to the dynamic real system, i.e. in our particular case, the values under the roots in Eq. (18) must be: $\frac{\alpha^{2}}{m^{2}}-\frac{2 c}{m}<0$, the roots $k_{1}$ and $k_{2}$ are complex numbers, and therefore, the general solution of homogeneous differential Eq. (16) will be as follows:

$$
Z_{\text {hom. }}=e^{-\frac{\alpha}{m} t}\left(C_{1} \sin \sqrt{\frac{\alpha^{2}}{m^{2}}-\frac{2 c}{m}} \cdot t+C_{2} \cos \sqrt{\frac{\alpha^{2}}{m^{2}}-\frac{2 c}{m}} \cdot t\right)
$$

where:

$C_{1}$ and $C_{2}$ - arbitrary constants

A particular solution $Z^{*}$ of inhomogeneous differential Eq. (14) will be sought in the following form:

$$
Z^{*}=M \cdot \sin \frac{2 \pi V t}{l_{1}}+N \cdot \cos \frac{2 \pi V t}{l_{1}}+R
$$

where:

$M, N, R$ - unknown coefficients

These coefficients are discovered by the method of undetermined coefficients. To do this, we perform a twofold differentiation of expression (20). We have:

$$
\begin{gathered}
\dot{Z}^{*}=M \frac{2 \pi V}{l_{1}} \cdot \cos \frac{2 \pi V}{l_{1}} t-N \frac{2 \pi V}{l_{1}} \cdot \sin \frac{2 \pi V}{l_{1}} t \\
\ddot{Z}^{*}=-M \frac{4 \pi^{2} V^{2}}{l_{1}^{2}} \cdot \sin \frac{2 \pi V}{l_{1}} t-N \frac{4 \pi^{2} V^{2}}{l_{1}^{2}} \cdot \cos \frac{2 \pi V}{l_{1}} t
\end{gathered}
$$

By substituting the expressions (20), (21) and (22) to differential Eq. (14), we obtain:

$$
\begin{aligned}
& -M \frac{4 \pi^{2} V^{2}}{l_{1}^{2}} \cdot \sin \frac{2 \pi V}{l_{1}} t-N \frac{4 \pi^{2} V^{2}}{l_{1}^{2}} \cdot \cos \frac{2 \pi V}{l_{1}} t+ \\
& +\frac{2 \alpha}{m}\left(M \frac{2 \pi V}{l_{1}} \cdot \cos \frac{2 \pi V}{l_{1}} t-N \frac{2 \pi V}{l_{1}} \cdot \sin \frac{2 \pi V}{l_{1}} t\right)+
\end{aligned}
$$

$$
\begin{gathered}
+\frac{2 c}{m}\left(M \cdot \sin \frac{2 \pi V t}{l_{1}}+N \cdot \cos \frac{2 \pi V t}{l_{1}}+R\right)= \\
=\frac{4 \pi \alpha h_{o} V}{m l_{1}} \cdot \sin \frac{2 \pi V}{l_{1}} t-\frac{2 c h_{o}}{m} \cdot \cos \frac{2 \pi V}{l_{1}} t+\frac{2 c h_{o}}{m}
\end{gathered}
$$

In expression (23), we compare the coefficients for the same functions, occurring in the left and right side of the expression above.

As a result, we obtain the following system of three algebraic linear equations related to the unknown $M, N$ and $R$ :

$$
\begin{gathered}
\left(\frac{2 c}{m}-\frac{4 \pi^{2} V^{2}}{l_{1}^{2}}\right) \cdot M-\frac{4 \pi \alpha V}{m l_{1}} \cdot N=\frac{4 \pi \alpha h_{o} V}{m l_{1}} \\
\frac{4 \pi \alpha V}{m l_{1}} \cdot M+\left(\frac{2 c}{m}-\frac{4 \pi^{2} V^{2}}{l_{1}^{2}}\right) \cdot N=-\frac{2 c h_{o}}{m} \\
\frac{2 c}{m} \cdot R=\frac{2 c}{m} h_{o}
\end{gathered}
$$

We determine from the last equation of system $R=h_{o}$.

The coefficients $M$ and $N$ are obtained from the first two equations (24) using the Cramer method.

We can find the main determinant of the system consisting of the coefficients of the first two equations of system (24), while $M$ and $N$ are unknown. We have:

$$
\begin{aligned}
\Delta & =\left|\begin{array}{cc}
\frac{2 c}{m}-\frac{4 \pi^{2} V^{2}}{l_{1}^{2}} & -\frac{4 \pi \alpha V}{m l_{1}} \\
\frac{4 \pi \alpha V}{m l_{1}} & \frac{2 c}{m}-\frac{4 \pi^{2} V^{2}}{l_{1}^{2}}
\end{array}\right|= \\
& =\left(\frac{2 c}{m}-\frac{4 \pi^{2} V^{2}}{l_{1}^{2}}\right)^{2}+\left(\frac{4 \pi \alpha V}{m l_{1}}\right)^{2}
\end{aligned}
$$

In next step, we calculate the determinant $\Delta_{M}$. It will be equal to:

$$
\Delta_{M}=\left|\begin{array}{cc}
\frac{4 \pi \alpha h_{o} V}{m l_{1}} & -\frac{4 \alpha \pi V}{m l_{1}} \\
-\frac{2 c h_{o}}{m} & \frac{2 c}{m}-\frac{4 \pi^{2} V^{2}}{l_{1}^{2}}
\end{array}\right|=-\frac{16 \pi^{3} \alpha V^{3} h_{o}}{m l_{1}^{3}}
$$

After that, we find the determinant $\Delta_{N}$. It will be equal to:

$$
\begin{gathered}
\Delta_{N}=\left|\begin{array}{cc}
\frac{2 c}{m}-\frac{4 \pi^{2} V^{2}}{l_{1}^{2}} & \frac{4 \pi \alpha h_{o} V}{m l_{1}} \\
\frac{4 \alpha \pi V}{m l_{1}} & -\frac{2 c h_{o}}{m}
\end{array}\right|= \\
=-\frac{2 c h_{o}}{m}\left(\frac{2 c}{m}-\frac{4 \pi^{2} V^{2}}{l_{1}^{2}}\right)-\frac{16 \pi^{2} \alpha^{2} V^{2} h_{o}}{m^{2} l_{1}^{2}}
\end{gathered}
$$

Using Cramer's rule, we find the coefficients $M$ and $N$ :

$$
M=\frac{\Delta_{M}}{\Delta}=-\frac{16 \pi^{3} \alpha V^{3} h_{o}}{m l_{1}^{3}\left[\left(\frac{2 c}{m}-\frac{4 \pi^{2} V^{2}}{l_{1}^{2}}\right)^{2}+\frac{16 \pi^{2} \alpha^{2} V^{2}}{m^{2} l_{1}^{2}}\right]}
$$




$$
N=\frac{\Delta_{N}}{\Delta}=-\frac{\frac{2 c h_{0}}{m}\left(\frac{2 c}{m}-\frac{4 \pi^{2} V^{2}}{l_{1}^{2}}\right)+\frac{16 \pi^{2} \alpha^{2} V^{2} h_{o}}{m^{2} l_{1}^{2}}}{\left(\frac{2 c}{m}-\frac{4 \pi^{2} V^{2}}{l_{1}^{2}}\right)^{2}+\frac{16 \pi^{2} \alpha^{2} V^{2}}{m^{2} l_{1}^{2}}}
$$

Substituting the expressions (28) and (29) to (20), we obtain a particular solution $Z^{*}$ of inhomogeneous differential Eq. (14). We have:

$$
\begin{aligned}
& Z^{*}=-\frac{16 \pi^{3} \alpha V^{3} h_{o}}{m l_{1}^{3}\left[\left(\frac{2 c}{m}-\frac{4 \pi^{2} V^{2}}{l_{1}^{2}}\right)^{2}+\frac{16 \pi^{2} \alpha^{2} V^{2}}{m^{2} l_{1}^{2}}\right]} \cdot \sin \frac{2 \pi V}{l_{1}} t- \\
& -\frac{\frac{2 c h_{o}}{m}\left(\frac{2 c}{m}-\frac{4 \pi^{2} V^{2}}{l_{1}^{2}}\right)+\frac{16 \pi^{2} \alpha^{2} V^{2} h_{o}}{m^{2} l_{1}^{2}}}{\left(\frac{2 c}{m}-\frac{4 \pi^{2} V^{2}}{l_{1}^{2}}\right)^{2}+\frac{16 \pi^{2} \alpha^{2} V^{2}}{m^{2} l_{1}^{2}}} \cdot \cos \frac{2 \pi V}{l_{1}} t+h_{o}
\end{aligned}
$$

Expression (30) defines the law of forced oscillations of the centre of mass of sensing (gauge) wheels of the sugar beet leaf harvester.

Taking into account the expressions (15), (19) and (20), we can write the general solution of differential Eq. (14):

$$
\begin{aligned}
Z=e^{-\frac{\alpha}{m} t} & \left(C_{1} \sin \sqrt{\frac{\alpha^{2}}{m^{2}}-\frac{2 c}{m}} \cdot t+C_{2} \cos \sqrt{\frac{\alpha^{2}}{m^{2}}-\frac{2 c}{m}} \cdot t\right)+ \\
& +M \cdot \sin \frac{2 \pi V}{l_{1}} t+N \cdot \cos \frac{2 \pi V}{l_{1}} t+R
\end{aligned}
$$

where:

$M, N$ and $R$ - are determined from expressions (28), (29) and $R=h_{\text {o }}$ respectively

The arbitrary constants of integration $C_{1}$ and $C_{2}$ are defined from the following initial conditions $t=0: Z=0$, $\dot{Z}=0$.

To do this we have to differentiate the expression (31) according to time. We have:

$$
\begin{aligned}
& \dot{Z}=-\frac{\alpha}{m} e^{-\frac{\alpha}{m} t}\left(C_{1} \sin \sqrt{\frac{\alpha^{2}}{m^{2}}-\frac{2 c}{m}} \cdot t+C_{2} \cos \sqrt{\frac{\alpha^{2}}{m^{2}}-\frac{2 c}{m}} \cdot t\right)+ \\
&+e^{-\frac{\alpha}{m} t} \cdot\left(C_{1} \sqrt{\frac{\alpha^{2}}{m^{2}}-\frac{2 c}{m}} \cdot \cos \sqrt{\frac{\alpha^{2}}{m^{2}}-\frac{2 c}{m}} \cdot t-C_{2} \sqrt{\frac{\alpha^{2}}{m^{2}}-\frac{2 c}{m}} \cdot \sin \sqrt{\frac{\alpha^{2}}{m^{2}}-\frac{2 c}{m}} \cdot t\right)+ \\
&+M \cdot \frac{2 \pi V}{l_{1}} \cos \frac{2 \pi V}{l_{1}} t-N \cdot \frac{2 \pi V}{l_{1}} \sin \frac{2 \pi V}{l_{1}} t
\end{aligned}
$$

By substituting the values of initial conditions to expressions (31) and (32), we obtain the unknown $C_{1}$ and $C_{2}$.

When substituting the values of constants $C_{1}$ and $C_{2}$ to expression (31), we finally find the general solution of differential Eq. (30):

$$
Z=e^{-\frac{\alpha}{m} t}\left(-\frac{\frac{\alpha}{m}(N+R)+\frac{2 \pi V}{l_{1}} M}{\sqrt{\frac{\alpha^{2}}{m^{2}}-\frac{2 c}{m}}} \cdot \sin \sqrt{\frac{\alpha^{2}}{m^{2}}-\frac{2 c}{m}} \cdot t-\right.
$$

$\left.-(N+R) \cdot \cos \sqrt{\frac{\alpha^{2}}{m^{2}}-\frac{2 c}{m}} \cdot t\right)+M \cdot \sin \frac{2 \pi V}{l_{1}} t+N \cdot \cos \frac{2 \pi V}{l_{1}} t+R$
The analytical expression (33) defines the law of translational vertical vibrations of the centre of mass of the sensing (gauge) wheels (point $B$ ).

During numerical simulation on the $P C$, the value of the forward speed of the tractor with sugar beet leaf harvester machine was changing from $V=2.5 \mathrm{~m} \cdot \mathrm{s}^{-1}$ to $V=3.8 \mathrm{~m} \cdot \mathrm{s}^{-1}$. There were also used different values of the moments of inertia $I_{\text {oy }}$ of the sugar beet leaf harvester (taking into account the weight of the sugar beet leaves inside the machine), which depend on $M$ - the weight of sugar beet leaf harvester and $L$ - the distance from the axis of the sensing (gauge) wheels to the suspension point $O$ (Fig. 2). During calculations, there were chosen several values of moments of inertia: from $I_{\text {oy }}=30 \mathrm{~kg} \cdot \mathrm{m}^{2}$ to $I_{\text {oy }}=60 \mathrm{~kg} \cdot \mathrm{m}^{2}$.

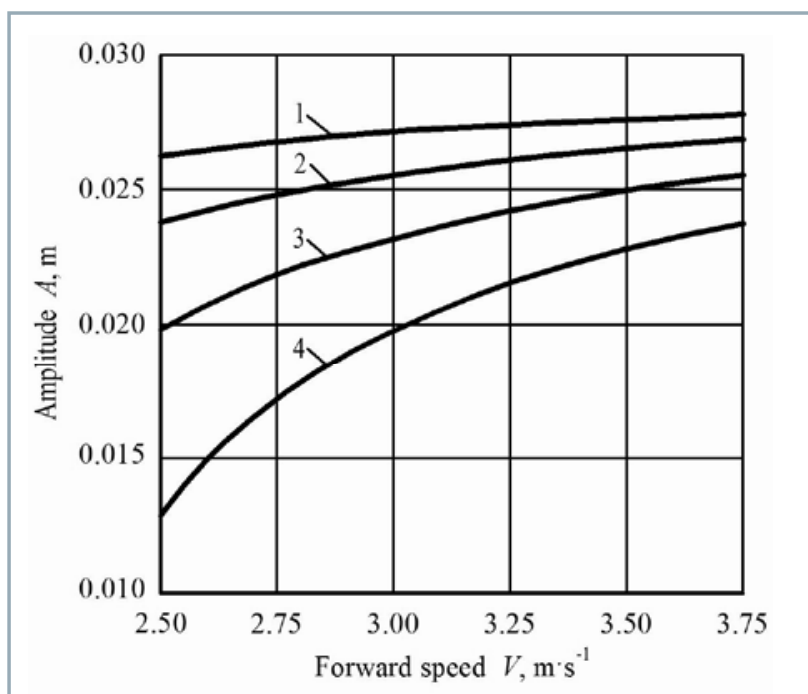

Fig. 3 Dependence of the amplitude $A$ of own vibrations of the gravity centre of sensing wheels of the sugar beet leaf harvester on machine forward speed $V$ for a different step of soil surface unevenness: $1-l_{1}=1.05 \mathrm{~m}$; $2-I_{1}=1.40 \mathrm{~m} ; 3-I_{1}=1.75 \mathrm{~m} ; 4-I_{1}=2.10 \mathrm{~m}$

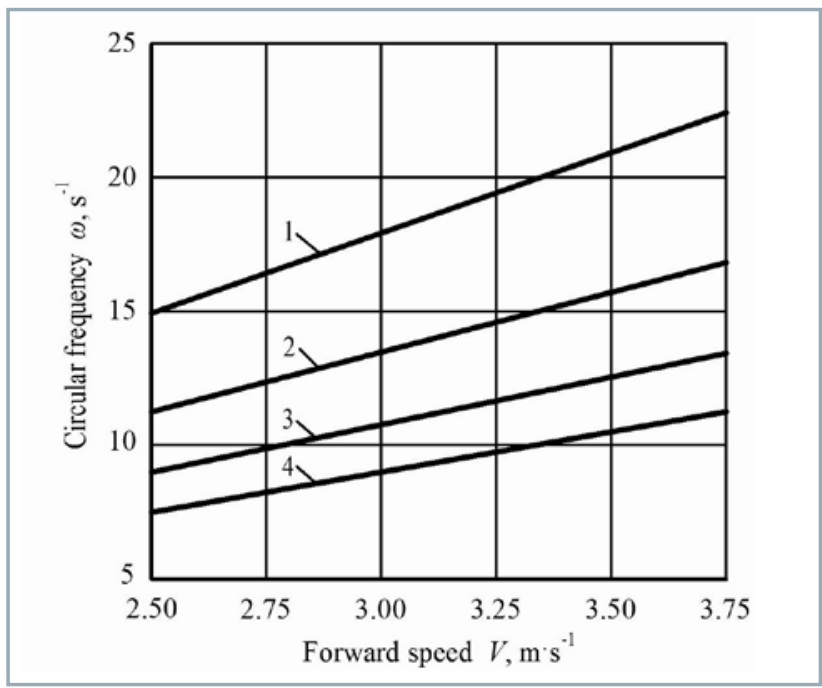

Fig. 4 Dependence of the frequency $\omega$ of constrained vibrations of the gravity centre of the sugar beet leaf harvest machine on forward speed $V$ for a different step of soil surface unevenness: $1-l_{1}=1.05 \mathrm{~m}$; $2-I_{1}=1.40 \mathrm{~m} ; 3-I_{1}=1.75 \mathrm{~m} ; 4-I_{1}=2.10 \mathrm{~m}$ 
The results of numerical modelling on the PC according to the own mathematical model of vibrations of the sugar beet leaf harvester front-mounted on a universal wheeltype tractor are presented in Fig. 3 and Fig. 4.

The analysis of the functions presented in Fig. 3 allows us to confirm that increasing of the working width of machines used for basic tillage in sugar beet growing system (which can be considered a source of cosinusoidal soil surface profile), as well as improving of the subsequent soil surface profile of the sugar beet field, are significantly lowering the vibrations of the front-mounted sugar beet leaf harvest machine, especially for forward speed up to $3.0 \mathrm{~m} \cdot \mathrm{s}^{-1}$.

From the functions presented in Fig. 4, it can be seen that the frequency of forced oscillations of the mass centre of the machine front-mounted on the tractor does not exceed $10 \mathrm{~s}^{-1}$ in the entire range of agro-technical forward speeds when using sugar beet leaf harvest machines with a working width of more than $2.2 \mathrm{~m}$.

Numerical modelling and obtained vibration characteristics of the front-mounted sugar beet harvester on the basis of universal wheel-type tractor (pulling power category 3) confirmed that, for the accepted constructivekinematic parameters, this oscillating system is capable of extinguishing perturbing influences caused by field surface. So, for example, at the forward speed of the sugar beet leaf harvest machine $V=3.5 \mathrm{~m} \cdot \mathrm{s}^{-1}$, the amplitude $A$ of own vibrations of the mass centre of the sugar beet leaf harvest machine copying wheels is reduced 2.2-2.7 times compared to the height of the field surface unevenness $h_{o}=0.06 \mathrm{~m}$.

\section{Conslusions}

1. A new machine and tractor unit, sugar beet leaf harvester front-mounted on universal wheel-type tractor, which provides flat cut of the basic mass of the sugar beet leaves within the all working width, was developed.

2. The sugar beet leaf harvester front-mounted on universal tractor, during its operation, creates angle vibrations in longitudinal vertical plane due to motion on uneven soil surface. These vibrations can be considered kinematic activators, as well as independent oscillatory motion due to elastic-damping properties of the pneumatic sensing (gauge) wheels moving between the rows of sugar beets and having appropriate stiffness and damping coefficients.

3. For the studied dynamical system, an equivalent scheme of movement in longitudinal vertical plane was developed with the identification of all forces acting on it, their size and design of the given system of coordinates, as well as a choice of generalized coordinates.

4. On the basis of the input equations of dynamics in the Lagrange II form, there was obtained a system of two nonlinear differential equations of oscillation of the sugar beet leaf harvester front-mounted on a wheeltype tractor. The abovementioned equation system is based on relation to relatively unknown and generalized coordinates $\varphi$ and $Z$; the system represents the estimated mathematical model of its motion.

5. Conducted analytical transformation made it possible: to obtain the general solution of differential equation; to find the law of translational vertical vibrations of the centre of mass of sensing (gauge) wheels when driving over the soil surface relief; to find the final expression of the amplitude of its own and forced oscillations of the centre of mass of gauge wheels of the sugar beet leaf harvester; as well as to observe the circular frequency and forced oscillations of these wheels.

6. Final analytical equation, allowing to obtain information on the vertical movement of any frame point (including lower and upper point of the rotary sugar beet leaf cutting mechanism) due to the sugar beet leaf harvester vibrations during its operation, was obtained for further numerical modelling of design and kinematic parameters of the sugar beet leaf harvester (front-mounted on a tractor).

7. Numerical modelling on a PC and obtained vibrations characteristics of the front-mounted sugar beet harvester on the basis of universal wheel-type tractor (pulling power category 3) allows us to determine the effects of forward speed regime on the amplitude of sugar beet leaf harvest machine vibrations.

\section{References}

BOSOJ, E. S. - VERNJAEV, O. V. - SMIRNOV, I. I. - SULTAN-SHAKH, E. G. 1978. Theory, Design and Calculations of Agricultural Machines. Textbook for universities of agricultural machinery. $2^{\text {nd }}$ edition, Moscow: Mashinostroenije, 568 pp. (In Russian: Teorija, konstrukcija, i raschot selskokhozjajstvennykh mashin).

BULGAKOV, V. M. - KORENKO, M. 2007. Mathematical model of sugar beet vibratory digging up. In Acta Technologica Agriculturae, vol. 10, no. 3-4, pp. 61-65.

BULGAKOV, V. M. - ADAMCHUK, V. V. - NOZDROVICKY, L. - BORIS, M. - IHNATIEV, Y. I. 2016. Properties of the sugar beet tops during the harvest. In Proceedings of 6th International Conference on Trends in Agricultural Engineering, 7-9 September 2016, Prague, Czech Republic, pp. 102-108.

BUTENIN, N. V. - LUNC, J. L. - MERKIN D. R. 1985. Course of Theoretical Mechanics. $2^{\text {nd }}$ part. Dynamics. Moscow: Nauka, 495 pp. (In Russian: Kurs teoreticheskoj mekhaniky. Tom 2. Dynamika).

HUIJBREGTS, T. - LEGRAND, G. - HOFFMANN, C. - OLSSON, R. OLSSON, A. 2013. Long-term storage of sugar beet in North-West Europe. COBRI report, 2013, no. 1, 54 pp.

IHNATIEV, Y. 2016. Theoretical substantiation of topping parameters without sugar beet head copying. In Mechanization in Agriculture, no. 2, pp. 10-12.

JOBBÁGY, J. - GABAJ, D. - ÁRVAY, J. 2011. Evaluation of selected agro-physical properties of a root vegetable. In Acta Technologica Agriculturae, vol. 14, no. 3, pp. 61-66.

KHVOSTOV, V. A. - REJNGART, E. S. 1995. Machines for Harvest of Root Crops and Onion (Theory, Design and Calculations). Moscow: VISKHOM, 391 pp. (In Russian: Mashiny dlja uborky korneplodov i luka (teorija, konstrukcija i raschot)).

KUTKOV, G. M. 2004. Tractors and Lorries. Theory and Technological Properties. Moscow : Kolos, 504 pp. (In Russian: Traktory i avtomobily. Teorija i tekhnologicheskie svojstva).

LAMMERS, S. - OLAF, P. - OLAF, R. 2010. Defoliation of sugar beets - assessment of quality and gain in delivered beet mass. In Landtechnik, no. 6, pp. 464-467.

LILLEBOE, D. 2014. Optimizing defoliator \& harvester performance. In The Sugarbeet Grower, vol. 53, no. 6, pp. 6-13.

MOROZOV, B. I. - GRINGAUZ, N. M. 1969. Calculation of movement of wheeled machine on uneven road. In Mekhanizacija i Elektrifikacija of the Socialisticheskogo Selkhogo Khozjajstva, no 7, pp. 11-14. (In Russian: Raschot dvizhenija kolesnoj mashiny po nerovnoj doroge). VASILENKO, P. M. 1996. Introduction in Agricultural Mechanics. Kiev: Selkhozobrazovanie, 252 pp. (In Russian: Vvedenie v zemledelcheskuju mekhaniku). 